Check for updates

Cite this: RSC Adv., 2018, 8, 342

Received 13th October 2017

Accepted 7th December 2017

DOI: 10.1039/c7ra11300e

rsc.li/rsc-advances

\section{Polyhydroxy gemini surfactant as a mechano- responsive rheology modifier for inverted emulsion drilling fluid}

\author{
Yinbo He, (D) Guancheng Jiang, * Zhengqiang Deng, Fan Liu, Shuanglei Peng, \\ Xiaoxiao Ni, Yawei Shi and Wuge Cui
}

Due to particularity of inverted emulsion, inverted emulsion drilling fluid (IEDF) always has poor mechanoresponsive rheology, which greatly restricts its application in the petroleum industry. This study reports the effectiveness of a novel polyhydroxy gemini surfactant (PGS) in responsive rheology modification for inverted emulsion. PGS is prepared by amidation between dimer fatty acid and diethanolamine at a molar ratio of $1: 2$. Rheology tests show that PGS can effectively improve emulsion shear thinning and thixotropic performance as well as viscoelasticity. Modification with PGS is more significant for emulsion at higher volume fraction. Based on various investigations including morphology, stability, interfacial properties, and linear fitting of rheological data, reasonable deductions and probable mechanism are proposed. The interfacial accumulation of PGS makes interfacial film gel-like and droplets attractive. The sensitive attraction enables droplets to exhibit time-dependent and shear-sensitive flocculation depending on outer mechanical conditions, resulting in mechano-responsive rheology of emulsion. In addition to acting independently, PGS is also synergistic with organophilic clay, which suggests the potential use of PGS as a high-performance rheology modifier not only for clay-free, but also for traditional IEDFs

\section{Introduction}

Inverted emulsion drilling fluid (IEDF) is a type of highperformance oil-based drilling fluid which is based on inverted emulsion. ${ }^{1-3}$ Compared with water-based drilling fluid (WDF), ${ }^{4-6}$ IEDF possesses excellent stability, lubrication, inhibition, reservoir protection performance and tolerance for salinity and temperature. ${ }^{7}$ With these appealing features, IEDF has been applied worldwide in various challenging drilling operations, such as complex structural wells, ultra-deep water, and unconventional hydrocarbon reservoirs.

In technical considerations, the desirable rheology for drilling fluid is supposed to be responsive to outer mechanical conditions. ${ }^{8-10}$ Specifically, in flow state, drilling fluid has desirable shear thinning performance, so that the high-shearrate-viscosity (HSRV) is as low as possible, while the lowshear-rate-viscosity (LSRV) is sufficiently high. After shear stops, owing to its thixotropic properties, drilling fluid is able to form strong gel structures in short time. Such mechanoresponsiveness is very advantageous for rock-breaking, cutting-carrying, solid-suspending, borehole-cleaning and reducing circulating pressure loss. ${ }^{11,12}$ However, for IEDF, responsive rheology, which is determined by the particularity of

MOE Key Laboratory of Petroleum Engineering, China University of Petroleum, Beijing, 102249, China. E-mail: 15010025286@126.com the base fluid, in this case inverted emulsion, is difficult to achieve. ${ }^{13-15}$ Typically, oils used for IEDF such as diesel, paraffin or polyalphaolefin, are Newtonian fluids, which make the continuous phase of emulsion purely viscous, but never viscoelastic. In addition, to avoid instability, the volume fraction $(\phi)$ of water in emulsion used for IEDF is rather low. Therefore, the inter-droplet interaction of water droplets is weak, resulting in unsatisfactory responsive rheology of the emulsion. ${ }^{16,17}$

A traditional way to improve responsive rheology of IEDF is to use rheology modifiers. ${ }^{18,19}$ The most commonly-used modifier is organophilic clay (OC), which is prepared by adsorption of lipophilic cationic surfactants ${ }^{20}$ such as trimethylstearylammonium chloride on montmorillonite. Through selfassembly, OC accumulates into microstructures at oil/water interfaces and increases gel strength of inverted emulsion. ${ }^{21}$ Since these microstructures are physically-associated, rheology of IEDF can be modified responsively. However, under low loading, the performance of OC is relatively weak as a consequence of minimal electrical interaction between clay particles. ${ }^{22}$ In particular, similar to negative influences of bentonite overuse on WDF, OC overuse causes excessive viscosity and thick filtrate cake in IEDF. ${ }^{23}$ In addition to clay, polymer is considered an effective rheology modifier since it can interweave into sensitive macromolecular structures at low addition amounts. For example, water-soluble polymers such as polyelectrolytes are effective gelators to prepare oil-in-water 
emulsion gels..$^{15}$ However, for inverted emulsions, because of the strong apolarity of the continuous oil phase, such polymer gelators are quite scant and ineffective.

In general, the responsive rheology of fluids derives from internal responsive networks, which are driven by reversible interactions. ${ }^{9}$ It is well-proven that networks of emulsion can be constructed by physically-associated droplets. ${ }^{24}$ Furthermore, the tendency of droplets to form three-dimensional networks is closely related with properties of interfacial film. ${ }^{13}$ Thus, it is feasible to utilize functional surfactants to modify rheology of inverted emulsion responsively. ${ }^{25}$ Owing to special doubleamphiphilic molecular structure, gemini surfactants possess amazingly high surface activity, which greatly facilitates adsorption and interfacial modification. ${ }^{26}$ A number of gemini surfactants have been developed and utilized for emulsion polymerization,,$^{27}$ gelatinization, ${ }^{28,29}$ solubilization, ${ }^{30}$ delivery and oil displacement. ${ }^{31,32}$ To our knowledge, however, they have not yet been systematically investigated and used as mechanoresponsive rheology modifiers, particularly for inverted emulsion.

In this study, we prepared a special polyhydroxy gemini surfactant (PGS) and comprehensively demonstrated its responsive modification of the rheological properties of inverted emulsion. Based on various investigations, a probable mechanism of PGS's action is proposed. In addition, the synergistic effect between PGS and OC is investigated. The purpose of the study reported herein is to provide an innovative method to improve the mechano-responsiveness of IEDF, and further to develop a better understanding of the relation between interfacial properties and the responsive rheology of inverted emulsion.

\section{Materials and methods}

\subsection{Materials}

Highly purified dimer fatty acid (DFA; dimer content is over 98\%) was purchased from Croda Inc. Diethanolamine (DEA) and sorbitan monooleate (SPAN-80) were purchased from TCI Inc. Liquid paraffin (apparent viscosity at $1021.8 \mathrm{~s}^{-1}$ is 22.5 $\mathrm{mPa} \mathrm{s}$; density is $0.88 \mathrm{~g} \mathrm{~cm}^{-3}$; flash point is $185.0{ }^{\circ} \mathrm{C}$ ) was purchased from Arcos Organics Inc. OC (VG-Plus) was kindly provided by M-I SWACO Inc. All the chemicals were used without further purification.

\subsection{PGS preparation}

PGS was synthesized by amidation reaction..$^{33}$ DFA and DEA at molar ratio of $1: 2$ were accurately weighed and poured into a three-neck glass flask loaded with a blender, a water separator and a condenser. The blend was first mixed for $20 \mathrm{~min}$ and then heated to $170{ }^{\circ} \mathrm{C}$. The reaction was terminated after $3 \mathrm{~h}$ and the product was purified using a filtration membrane with molecular weight cutoff of $500 \mathrm{~g} \mathrm{~mol}^{-1}$.

\subsection{Structural characterization}

Proton nuclear magnetic resonance $\left({ }^{1} \mathrm{H}\right.$ NMR) spectroscopy analyses of DFA and PGS were performed using deuterated dimethyl sulphoxide (DMSO) on a JNM-ECA600 (JEOL Inc., Japan) spectrometer at resonance frequency of $600 \mathrm{MHz}$. Fourier transform infrared spectroscopy (FT-IR) analyses of DFA, DEA and PGS were conducted using Magna-IR560 spectrometer (Nicolet Inc., U.S.A) in the wavenumber range of $4000-400 \mathrm{~cm}^{-1}$.

\subsection{Emulsion preparation}

Inverted emulsions were prepared at $\phi$ of $0.2,0.3,0.4$ and 0.5 using a high-speed homogenizer. The total volume of paraffin and deionized water was $300 \mathrm{~mL}$ and the addition of SPAN-80 was $15 \mathrm{~g}$ in each emulsion. Emulsions were prepared under the same procedure in order to obtain approximately equal mean droplet size and polydispersity. First, the blend of paraffin and SPAN-80 was mixed at $3000 \mathrm{rpm}$ for $5 \mathrm{~min}$. Then the rotation speed was increased to $10000 \mathrm{rpm}$ and water was added dropwise. After water was completely incorporated into paraffin, the emulsion was homogenized at $12000 \mathrm{rpm}$ for $1 \mathrm{~h}$. Emulsions with PGS and OC were prepared by adding those components into the prepared emulsions and mixing at $12000 \mathrm{rpm}$ for $20 \mathrm{~min}$. The loading of PGS and OC was calculated depending on weight of water and weight of total emulsion respectively.

\subsection{Rheology}

HAAKE MARS60 rheometer (Thermo Fisher Scientific Inc., U.S.A) was used to investigate rheology of emulsion at $25{ }^{\circ} \mathrm{C}$. The rheometer was operated under plate model using C35 $1^{\circ} / \mathrm{Ti}$ rotor. Sample volume was $0.2 \mathrm{~mL}$ and gap distance was 0.053 $\mathrm{mm}$. To avoid influences of demulsification on rheology, all the tests were performed using freshly-prepared emulsions and finished in relatively short time.

Shear thinning performance was evaluated from the apparent viscosity-shear rate curve, which was obtained under stepwise rotation model. The shear rate was set from $1000 \mathrm{~s}^{-1}$ to $1 \mathrm{~s}^{-1}$ in 30 steps logarithmically. The steady apparent viscosity was recorded under each shear rate.

Thixotropic loop was obtained under continuous rotation model. Sample was sheared under shear rates ranging from $1 \mathrm{~s}^{-1}$ to $500 \mathrm{~s}^{-1}$ and from $500 \mathrm{~s}^{-1}$ to $1 \mathrm{~s}^{-1}$ in $20 \mathrm{~min}$ without pauses.

Gel recovery was evaluated under a combined shear-pauseoscillation model. Sample was first sheared at $25000 \mathrm{~s}^{-1}$ for $5 \mathrm{~min}$. Then, the shear was paused for a certain recovery time. Finally, a quick oscillation lasting $0.2 \mathrm{~min}$ at $10 \mathrm{~Hz}$ was exerted and the average elastic modulus $G^{\prime}$ was calculated. All the oscillatory measurements were performed with $1 \%$ strain since the strain sweep test showed that it was well within the linear viscoelastic region.

Viscoelasticity was evaluated under low-amplitudeoscillation frequency sweep model. Oscillation frequency was increased from $0.01 \mathrm{~Hz}$ to $10 \mathrm{~Hz}$ logarithmically and the corresponding elastic modulus $G^{\prime}$ and viscous modulus $G^{\prime \prime}$ were recorded. 


\subsection{Synergistic effect between PGS and OC}

A Fann model 35-A rotational viscometer (Fann Inc., U.S.A) was used to investigate the rheology of emulsion loaded with OC under the Bingham model. Tests were carried out in accordance with the American Petroleum Institute (API) guidelines. The stable viscosity readings were taken at rotations of $600 \mathrm{rpm}$ $\left(\phi_{600}\right), 300 \mathrm{rpm}\left(\phi_{300}\right), 6 \mathrm{rpm}\left(\phi_{6}\right)$ and $3 \mathrm{rpm}\left(\phi_{3}\right)$. Apparent viscosity (AV) was calculated as $\phi_{600} / 2$. Plastic viscosity (PV) was calculated as $\phi_{600}-\phi_{300}$. Yield point (YP) was calculated as $0.51 \times\left(\phi_{300}-\mathrm{PV}\right)$. Gel strength was measured as follows: the viscometer was initially operated at $600 \mathrm{rpm}$ for $1 \mathrm{~min}$, and then shut off for a certain time. Then, the value of gel strength was obtained by reading $\phi_{3}$ maximum. The unit of gel strength was transformed from $1 \mathrm{bf} / 100 \mathrm{ft}^{2}$ to $\mathrm{Pa}$ by multiplying by 0.48 .

\subsection{Interfacial properties}

Interfacial tension $\sigma$ of interfaces between paraffin and water with different loadings of SPAN-80 and PGS was tested using DTCA21 spinning drop tensiometer (Data Physics Inc., Germany) at $25{ }^{\circ} \mathrm{C}$.

Interfacial viscoelasticity of paraffin/water interface, paraffin + SPAN-80/water interface and paraffin + SPAN-80/water + PGS interface was evaluated using MCR301 interfacial rheometer (Anton Paar Inc., Austria) at $25{ }^{\circ} \mathrm{C} .{ }^{34} \mathrm{~A}$ bicone-type rotor was used to find the paraffin/water interface through the following procedures: first $100 \mathrm{~mL}$ water phase was slowly poured into the cylindrical container; then, the rotor moved vertically until the boundary between two cones was settled accurately at the water surface; finally, $50 \mathrm{~mL}$ paraffin phase was poured on water phase carefully. Oscillation frequency was increased from $0.01 \mathrm{~Hz}$ to $10 \mathrm{~Hz}$ logarithmically, and the corresponding interfacial complex modulus $\left|G^{*}\right|$, elastic modulus $G^{\prime}$ and viscous modulus $G^{\prime \prime}$ were recorded.

\subsection{Morphology}

The morphology of PGS in aqueous solution was observed by cryo-TEM on JEM-1400 transmission electron microscope (JEOL Inc., Japan) at $-174.8{ }^{\circ} \mathrm{C}$. BX41 optical microscope (Olympus Inc., Japan) was used to observe microscopic morphology changes of emulsion after the standing time. Samples were vigorously sheared at $25000 \mathrm{~s}^{-1}$ before each standing. After standing for certain time, samples were quickly settled on the microscope and observed.

\subsection{Stability}

Turbiscan-LAB stability analyser (Formulaction Inc., France) was used to evaluate static stability of emulsion at $25{ }^{\circ} \mathrm{C} .{ }^{35}$ The sample was settled vertically in a transparent glass tube and scanned every hour by a beam of light with a wavelength of $880 \mathrm{~nm}$. After each scan, the thickness of the upper clear layer was calculated as a percentage.

\section{Results and discussion}

\subsection{Characterizations of PGS}

DFA is an unsaturated binary acid with two carboxyl groups attached on a hydrocarbon chain consisting of 34 carbon atoms. The types of DFA isomers can be divided into acyclic, monocyclic and bicyclic depending on the different molecular structures of unsaturated bonds comprising the alkyl chain (Fig. 1). Since all the isomers can be observed as two fatty acid molecules linked by a $\mathrm{C}-\mathrm{C}$ covalent bond located between two $\mathrm{C}_{7} \mathrm{H}_{14} \mathrm{COOH}$ groups, DFA is an ideal compound to prepare gemini surfactants.

The acyclic structure was used to analyze the ${ }^{1} \mathrm{H}$ NMR spectra of DFA and PGS, which are presented in Fig. 2a and b, respectively, as the acyclic isomer is the most abundant among the three isomers. ${ }^{36}$ The distinct resonance observed for the carboxyl group of DFA (signal $\mathrm{h}$ at $11.88 \mathrm{ppm}$ ) disappears in Fig. $2 \mathrm{~b}$, while resonance for hydroxyl group of DEA clearly observed (signal k at $4.04 \mathrm{ppm}$ ). Signals related to the $\mathrm{CH}_{2}$ of DEA are observed in Fig. 2b at 3.27 ppm (signal i) and $3.41 \mathrm{ppm}$ (signal j). Moreover, after amidation, it is noted that the signal related to $\mathrm{CH}_{2}$ groups, located next to the carbonyls of DFA (signal $\mathrm{g}$ at $2.10 \mathrm{ppm}$ ), shifts to lower field and divides into multiple peaks (signal $\mathrm{g}^{\prime}$ at $2.30 \mathrm{ppm}$ ) due to stronger deshielding effect of newly-formed tertiary amide. The significant structural changes are reflected in the FT-IR spectra (Fig. 2c) as well. The main characteristic adsorption peaks of PGS are blunt stretching band of $\mathrm{OH}\left(3380.6 \mathrm{~cm}^{-1}\right)$, stretching bands of $\mathrm{C}-\mathrm{N}$ and $\mathrm{C}-\mathrm{O}\left(1066.4 \mathrm{~cm}^{-1}\right.$ and $\left.860.1 \mathrm{~cm}^{-1}\right)$, which arise from DEA, and rocking band of $\left(\mathrm{CH}_{2}\right)_{n}\left(723.2 \mathrm{~cm}^{-1}\right)$, which originate from DFA. In comparison with DFA, the stretching band of the $\mathrm{C}=\mathrm{O}$ of PGS red-shifts from $1708.6 \mathrm{~cm}^{-1}$ to $1621.9 \mathrm{~cm}^{-1}$ and the broad stretching band of $\mathrm{OH}$ on the carboxy group (around $3000 \mathrm{~cm}^{-1}$ ) disappears, indicating the formation of tertiary amide. These results demonstrate the desired reaction between DFA and DEA at mole ratio of $1: 2$ and thus the gemini structure of PGS. In addition, it is worth mentioning that the IR spectrum of PGS shows a weak adsorption at $1739.5 \mathrm{~cm}^{-1}$, which is attributed to the stretching band of $\mathrm{C}=\mathrm{O}$ in an ester group, while the typical strong adsorption peak of $\mathrm{C}-\mathrm{O}-\mathrm{C}$ stretching vibration of ester is not shown. This implies that minimal esterification could occur probably due to DFA impurity.

In Fig. 2c, similar associated stretching and deformation bands of hydroxyls in DEA and PGS are observed, indicating that PGS is highly-associative. PGS is well-dispersed in water and forms a optical creamy-white solution. In the TEM image shown in Fig. 2d, it is observed that PGS assembles into aggregated unilamellar vesicles with mean diameter of about $300 \mathrm{~nm}$, indicating its high surface activity.

\subsection{Responsive rheology modification of PGS on inverted emulsion}

The dependence of apparent viscosity on shear rate of pure emulsions and emulsions with PGS at different $\phi$ is presented in Fig. 3a. It is known that inverted emulsion is shear-thinned 


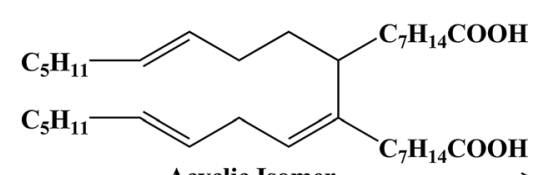

Acyclic Isomer
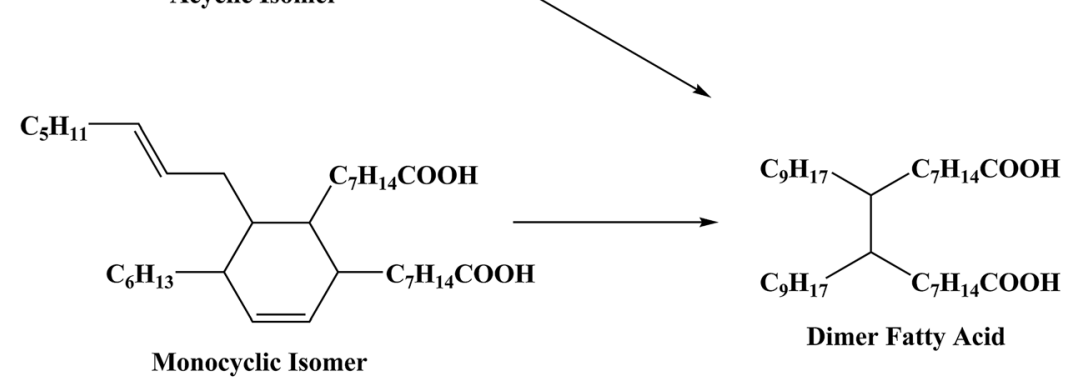

Dimer Fatty Acid

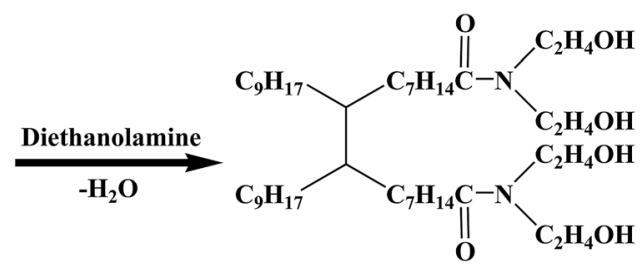

Polyhydroxy Gemini Surfactant

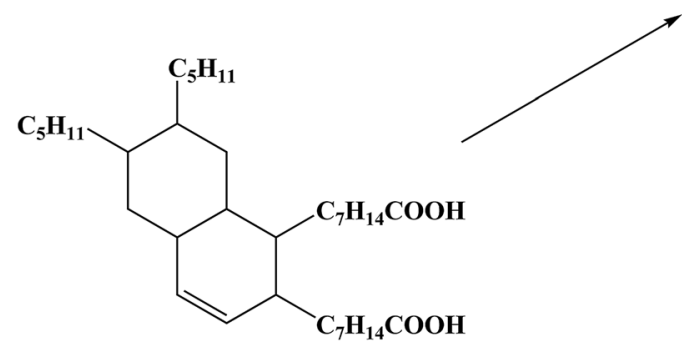

Bicyclic Isomer

Fig. 1 Molecular structures of DFA isomers (acyclic, monocyclic, bicyclic) and PGS.

because the viscosity decreases with the growth of shear rate. Since all the rheology curves do not show Newtonian plateaus within the test scopes, in order to make mathematical comparisons, flow curves are fitted under the power-law model, which is expressed logarithmically as

$$
\log \eta=\log K+(n-1) \log \gamma,
$$

where $\eta$ is the apparent viscosity, $K$ is the consistency coefficient, $n$ is the power law exponent and $\gamma$ is the shear rate. ${ }^{13}$ From eqn (1), it is known that lower $n$ indicates better shear thinning performance since shear thinning can be evaluated by the extent of change of apparent viscosity with shear rate. From values of $n$, it is found that emulsions with higher $\phi$ exhibit better shear thinning performance (Fig. $3 \mathrm{~b}$ ).

With the addition of PGS (Fig. 3a) it can be observed that for emulsions at any $\phi$, viscosity is increased and shear thinning is improved. The contribution of PGS to viscosity is related to shear rate, namely, the rise of HSRV brought by PGS is much lower than the rise of LSRV. Another noticeable phenomenon is that the modification of PGS on shear thinning is $\phi$-related, which is expressed as the slope of the log-log plot. Although the double-logarithmic plot could not perfectly linear, the slope helps in comparing data. ${ }^{37}$ As shown in Fig. $3 \mathrm{~b}$, with the growth of $\phi$, with the same loading of PGS, the decrease of $n$ becomes more significant, indicating more effective modification of PGS on emulsion.

To evaluate the influence of PGS on the thixotropy of inverted emulsions, a thixotropic loop test was applied. In Fig. 4a, it can be observed that the down curves of pure emulsions at $\phi$ of 0.2 , 0.3 and 0.4 do not show apparent lag behind up curves, indicating no considerable thixotropy. Pure emulsion at $\phi$ of 0.5 shows definite thixotropy, but the thixotropic energy is low since the loop area $A$ is small. Clearly, high $\phi$ can provide emulsion shear-sensitivity, but not much thixotropy. After PGS addition (Fig. 4b) the hysteresis becomes pronounced. The thixotropic energy of emulsions at different values of $\phi$ increases to varying extents. From the changes in loop areas, it can be said that the improvement of PGS on thixotropy is $\phi$ related. With the same loading of PGS, the emulsion at higher $\phi$ achieves better modification.

Technically, progressive gelation under static state is very important for drilling fluid. The quickly-formed networks enable drilling fluid to keep solids in suspension for a short time after the shear stops. In order to investigate the effect of PGS on gel recovery of emulsion, the dependence of recovered elastic modulus $G^{\prime}$ on standing time was tested. Before each standing, the emulsion was vigorously sheared to fully break gel structures inside. As shown in Fig. 5, $G^{\prime}$ of pure emulsion basically remains constant with standing time. In contrast, PGSloaded emulsion exhibits a noticeable gel recovery process, in which $G^{\prime}$ increases with the increase in time. Although the recovered $G^{\prime}$ could not completely represent the true gel strength because of the vigorous shear, the different timedependence of gel networks in pure emulsion and in PGSloaded emulsion is effectively demonstrated. Furthermore, it is observed that after the removal of shear, the instantaneous $G^{\prime}$ values of pure emulsion and PGS-loaded emulsion at the same $\phi, 0.5$, are similar. Nevertheless, in $5 \mathrm{~min}$, the $G^{\prime}$ of the latter almost doubles. From this aspect, PGS can progressively gelatinize emulsion without much tackifying effect. 

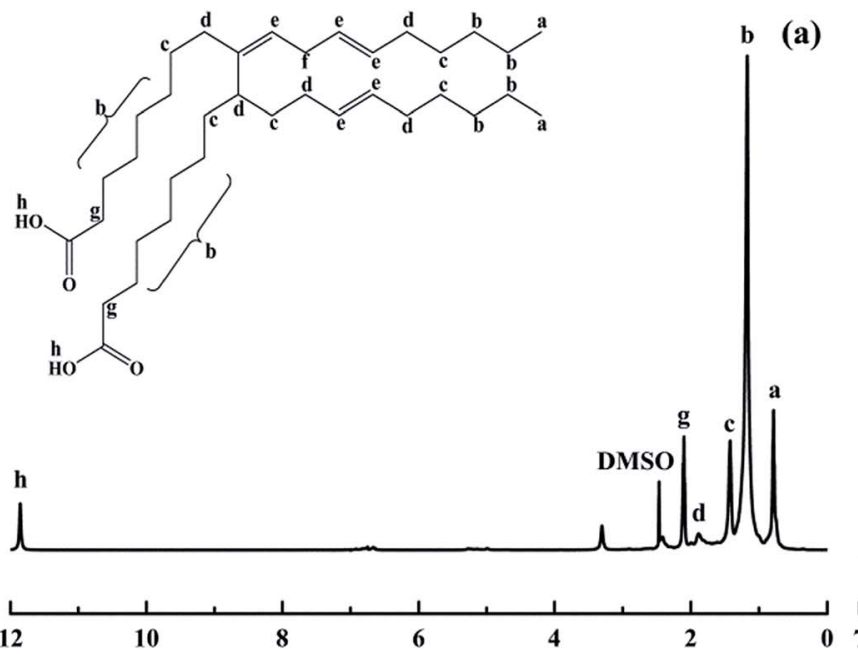

$\delta / p p m$
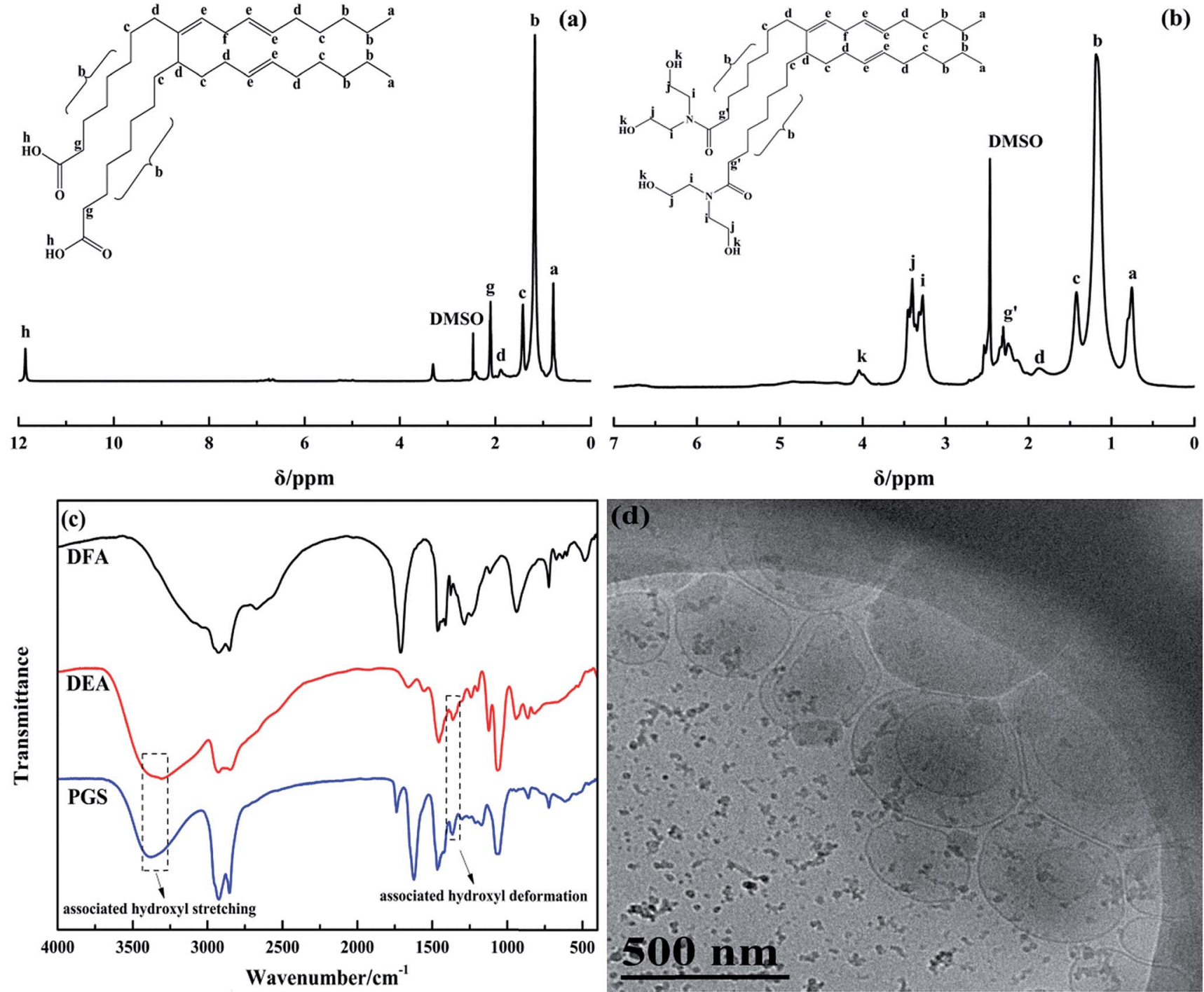

Fig. 2 Characterizations of PGS: (a) ${ }^{1} \mathrm{H}$ NMR spectrum of DFA; (b) ${ }^{1} \mathrm{H}$ NMR spectrum of PGS; (c) FT-IR spectra of DFA, DEA and PGS; (d) cryo-TEM image of PGS (0.5 wt\% in deionized water).

Based on the rheological data discussed above, it is known that PGS effectively improves shear thinning and thixotropic performance of inverted emulsion at a low addition amount. Moreover, to further investigate the modification of PGS on gel properties of emulsion, dynamic viscoelasticity was evaluated. From elastic modulus $G^{\prime}$ presented in Fig. 6, it is found that at a certain frequency, the growth of $G^{\prime}$ transforms from frequency-dependent to minor progressive. ${ }^{16,38}$ Beyond this critical frequency, $G^{\prime}$ basically stays constant, implying that emulsion acts like an elastic solid. ${ }^{38}$ Such transition occurs at approximately $1.47 \mathrm{~Hz}, 0.10 \mathrm{~Hz}, 0.05 \mathrm{~Hz}$ and $0.03 \mathrm{~Hz}$ for pure emulsions at $\phi$ of $0.2,0.3,0.4$ and 0.5 , respectively, exhibiting a declining tendency. Since emulsions at higher $\phi$ behave more like solids, $G^{\prime}$ can completely keep up with frequency growth in a wider range. After PGS addition, the frequency range, in which emulsion maintains progressive growth, becomes even wider. $G^{\prime}$ of PGS-loaded emulsions at $\phi$ of 0.4 and 0.5 does not show frequency-dependent growth in the entire test scope (Fig. 6b).
Moreover, compared with pure emulsions, emulsions with PGS definitely have higher $G^{\prime}$ values. Thus, it can be inferred that PGS strongly gelatinizes inverted emulsion.

Another remarkable phenomenon, as shown in Fig. 6, is that before increasing rapidly, $G^{\prime \prime}$ first decreases at intermediate frequencies and reaches a minimum. The minimum of $G^{\prime \prime}$ reduces after the addition of PGS, which is indicative of slow relaxation resulting from collective slipping motion of droplets. ${ }^{14}$ Apparently, droplets of PGS-loaded emulsion are more dynamic than those of pure emulsions. Since non-Newtonian rheology of emulsion directly depends on droplet dynamics, it is reasonable to conclude that PGS makes droplets more shearsensitive and time-dependent.

\subsection{Time-dependent droplets flocculation}

From the gel recovery test, it is deduced that pure emulsion and PGS-loaded emulsion exhibit totally different time-dependent 

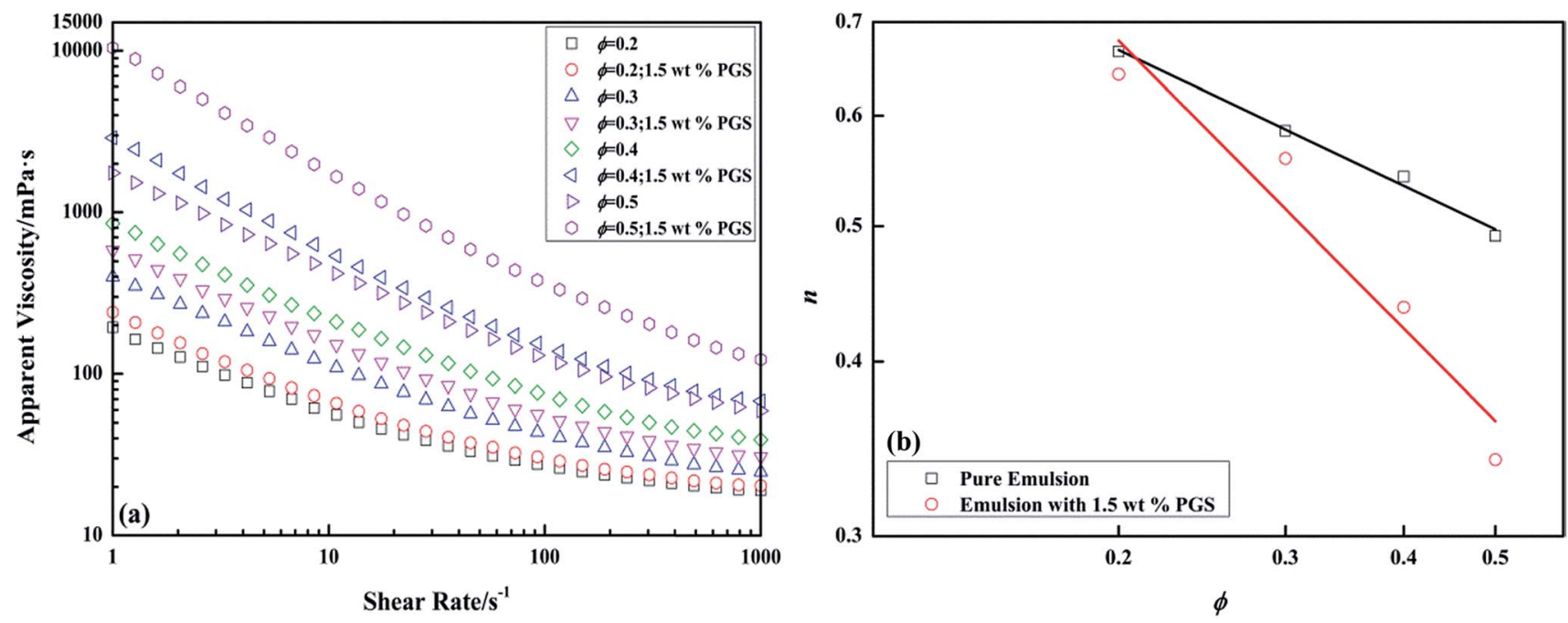

Fig. 3 Apparent viscosity-shear rate curves of pure emulsions and emulsions with 1.5 wt\% PGS at different $\phi$ (a) and variations of power law exponent $n$ with $\phi$ (b).
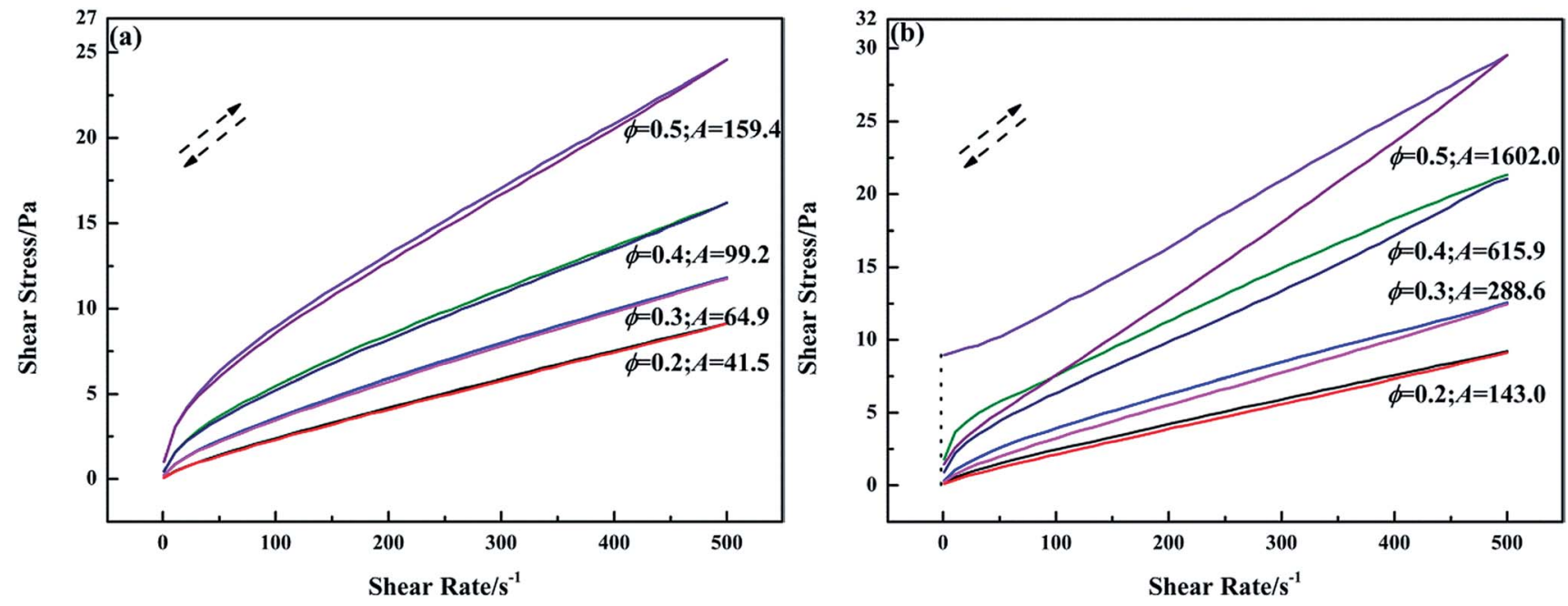

Fig. 4 Thixotropic loops of pure emulsions (a) and emulsions with 1.5 wt\% PGS (b) at different $\phi$ measured in shear rate range of $1-500 \mathrm{~s}^{-1}$.

behaviors. As gel formation is always accompanied by characteristic microscopic morphologies, we observed morphology changes of emulsion with time. As expected, it can be observed in Fig. $7 \mathrm{a}-\mathrm{c}$ that pure emulsion does not show visible microscopic changes with standing time. Droplets disperse independently all along owing to repulsion between emulsifier barriers. In contrast, PGS-loaded emulsion shows distinctive time-dependent morphology changes. It can be observed in Fig. $7 \mathrm{~d}$ that after standing for $10 \mathrm{~s}$, some droplets flocculate instead of independently dispersing. With increasing standing time, droplet flocculation becomes more evident. After standing for $30 \mathrm{~s}$, droplet clusters are clearly observed (Fig. 7e). After standing for $5 \mathrm{~min}$ (Fig. 7f) it can be observed that the majority of droplets are adhered closely, resulting in the formation of regional spanning networks. ${ }^{9}$ Based on such time-dependent changes, it can be concluded that after vigorous shear removal, the instantaneous droplet state of PGS-loaded emulsion should be independently-dispersed without flocculation.

The recovered $G^{\prime}$ values corresponding to Fig. $7 \mathrm{~d}-\mathrm{f}$ are 7.38 Pa, 8.75 Pa and 11.18 Pa, respectively (Fig. 5), exhibiting an increasing tendency. Obviously, droplet flocculation contributes to the gel strength of emulsion. In terms of rheology, ${ }^{\mathbf{1 6}}$ compared with independent droplets, flocculated droplets will produce stronger resistance under oscillation, since the deformation of adhered droplets creates additional interfaces and increases interfacial energy.

The time-dependent droplet flocculation is reflected in emulsion stability. As presented in Fig. 8a, well-emulsified pure emulsion at $\phi$ of 0.2 basically remains stable for $24 \mathrm{~h}$ without significant separation. After PGS addition, the height of the upper clear layer of emulsion increases sharply, which is ascribed to severe droplet flocculation and sedimentation..$^{39}$ The 


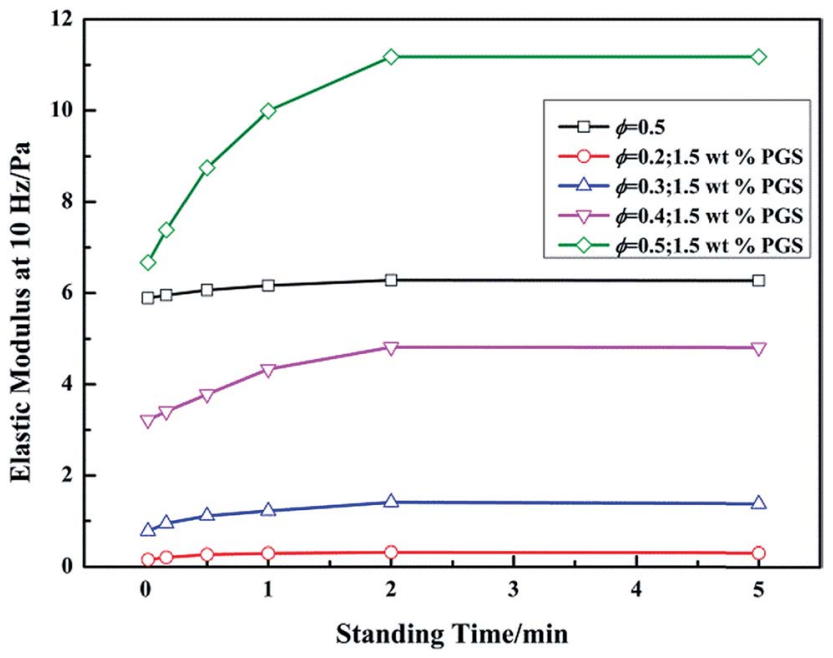

Fig. 5 Recoveries of elastic modulus $G^{\prime}$ (measured at $10 \mathrm{~Hz}$ ) of pure emulsion at $\phi$ of 0.5 and emulsions with $1.5 \mathrm{wt} \%$ PGS at $\phi$ of $0.2,0.3$, 0.4 and 0.5 with standing time.

phase separation is accelerated with PGS loading. For emulsion at high $\phi$, the results are opposite. In Fig. $8 \mathrm{~b}$, it is observed that the stability of emulsion at $\phi$ of 0.5 is improved by PGS. This phenomenon is the consequence of the spanning networks assembled by droplets, which improve suspension and thus make emulsion more stable. In contrast, droplets of PGS-loaded emulsion at low $\phi$ do flocculate, but could not create large spanning networks mostly due to insufficient droplet quantity.

It should be noted that both pure emulsions and PGS-loaded emulsions are polydispersed, which is ascribed to conventional emulsification process. ${ }^{40}$ For example, droplet diameter of pure emulsion at $\phi$ of 0.5 ranges from about $0.5 \mu \mathrm{m}$ to $3 \mu \mathrm{m}$ (Fig. 7). Although it has been proven that droplets with different sizes tend to flocculate, ${ }^{24}$ the flocculation phenomenon is not observed in polydispersed pure emulsions. In addition, in
Fig. 7, it is observed that PGS does not significantly change the mean size and polydispersity of droplets. Thus, it can be known that droplets of PGS-loaded emulsion are naturally prone to flocculate. In other words, PGS converts droplets from repulsive to attractive.

\subsection{Shear-sensitivity of inter-droplet interaction}

The inter-droplet interaction between repulsive deformable droplets strengthens with the decrease of inter-droplet distance. For pure emulsions at high $\phi$, the droplets strongly interacted with each other as they are close together. Thus, compared with emulsions at low $\phi$, emulsions at high $\phi$ would produce more resistance when they are deformed under shear, resulting in higher viscosity. ${ }^{13}$ The impact of inter-droplet interaction on emulsion viscosity is sensitive to shear. As demonstrated in Fig. 3a, HSRVs are always lower than LSRVs. This is because when shear rate increases, inter-droplet interaction is weakened owing to intense flow of interfacial film and decreased repulsion between emulsifiers. ${ }^{17}$ Since non-Newtonianism of pure emulsion strengthens with the growth of $\phi$, the emulsion at higher $\phi$ exhibits better shear thinning performance (Fig. 3b). ${ }^{\mathbf{1 4}}$

As mentioned above, the addition of PGS makes droplets attractive. Although it is rather difficult to directly observe the morphology of PGS-loaded emulsion in flowing state, it is reasonable to consider that flocculation exists under low shear due to weak mechanical disturbance. Similar to low-amplitudeoscillation, low shear also makes flocculated-droplets create additional interfaces and leads to higher resistance. Consequently, LSRVs are effectively increased by PGS. Under high shear, droplet flocculation is broken by intense mechanical forces and therefore the increase of HSRVs is not obvious. Under extreme high shear (25000 $\left.\mathrm{s}^{-1}\right)$, when interaction between droplets is negligible, as shown in Fig. 9, viscosities of PGS-loaded emulsions are only slightly higher than viscosities of pure emulsions. Based on such sensitive influences, the modification of PGS on shear thinning is achieved.
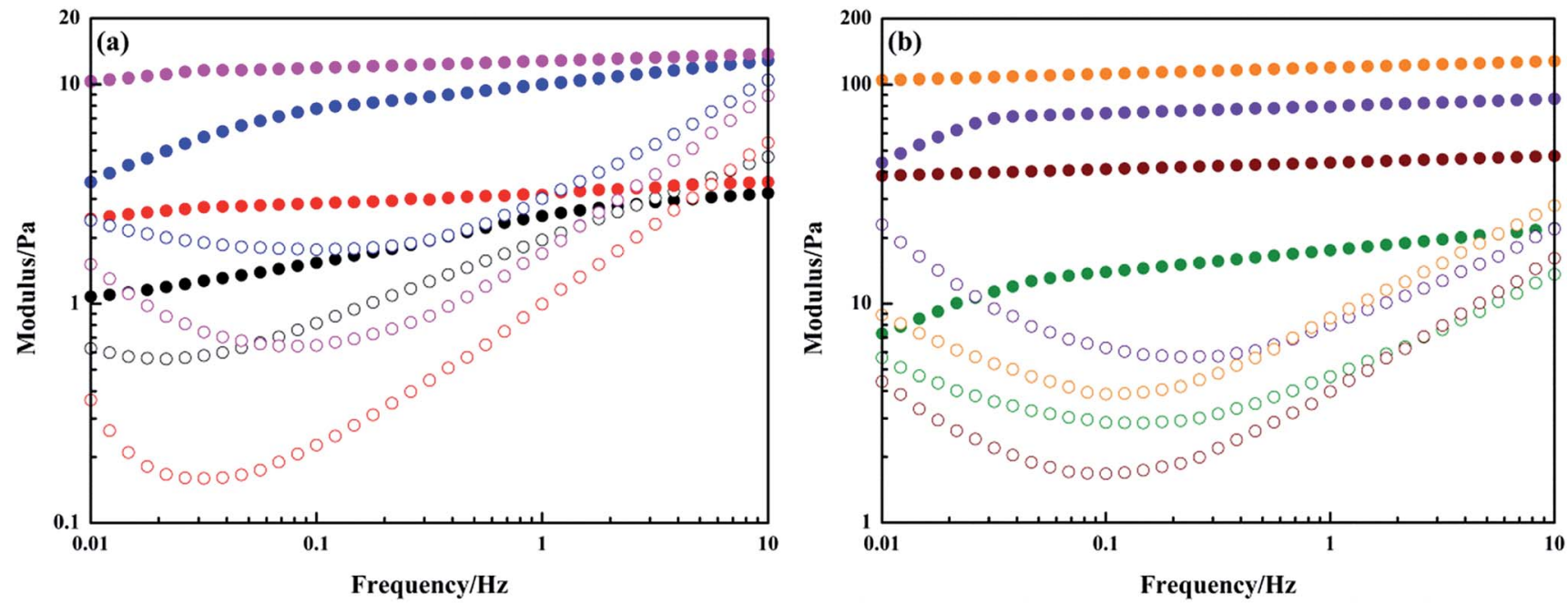

Fig. 6 (a, b) Frequency dependencies of elastic modulus $G^{\prime}$ (solid symbols) and viscous modulus $G^{\prime \prime}$ (open symbols) of pure emulsions at $\phi$ of 0.2 (black), 0.3 (blue), 0.4 (olive), 0.5 (violet) and emulsions with 1.5 wt\% PGS at $\phi$ of 0.2 (red), 0.3 (magenta), 0.4 (wine), 0.5 (orange). 

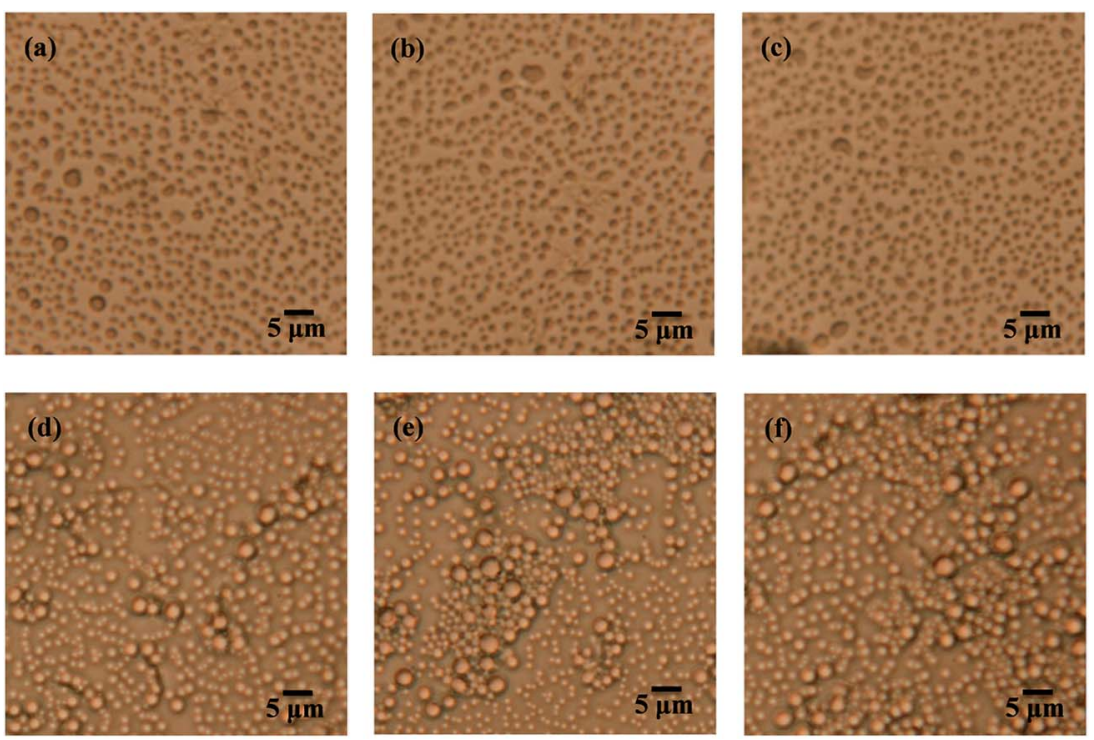

Fig. 7 Morphologies of pure emulsion and emulsion with $1.5 \mathrm{wt} \%$ PGS at $\phi$ of 0.5 at different standing times after vigorous shear: (a) pure emulsion after standing for $10 \mathrm{~s}$; (b) pure emulsion after standing for $30 \mathrm{~s}$; (c) pure emulsion after standing for 5 min; (d) PGS-loaded emulsion after standing for $10 \mathrm{~s}$; (e) PGS-loaded emulsion after standing for $30 \mathrm{~s}$; (f) PGS-loaded emulsion after standing for 5 min.

The rheology tests demonstrated that PGS is more effective for emulsions at high $\phi$. To investigate this $\phi$-related performance, viscosities measured at different shear rate are plotted with $\phi$ logarithmically. From Fig. 9, it can be observed that when inter-droplet interaction is negligible, namely, under extreme high shear, the $\phi$-related effect disappears. Slopes of pure emulsions and PGS-loaded emulsions are almost the same. With the decrease of shear rate, the difference between slopes rises and the $\phi$-related effect emerges. Apparently, the $\phi$-related effect is influenced by inter-droplet interaction. Noting that the main difference between PGS-loaded emulsions at different values of $\phi$ is droplet quantity, it is logical to attribute the $\phi$ related effect to the efficiency of droplet interaction. Despite the fact that droplets of emulsions loaded with equivalent PGS have almost the same propensity to flocculate, under low shear, the proportion of flocculated droplets in emulsion at high $\phi$ should be more than that in emulsion at low $\phi$, which is ascribed to larger droplet quantity and thus shorter inter-droplet distance. Therefore, though flocculation is broken under high shear, droplets of PGS-loaded emulsion at high $\phi$ are still more prone to interact with each other. Consequently, under extreme high shear, the $\phi$-related effect is no longer evident as the result of negligible inter-droplet interaction.

\subsection{Interfacial properties}

Considering the water-solubility and surface activity of PGS, it is reasonable to correlate the rheological modification of PGS on
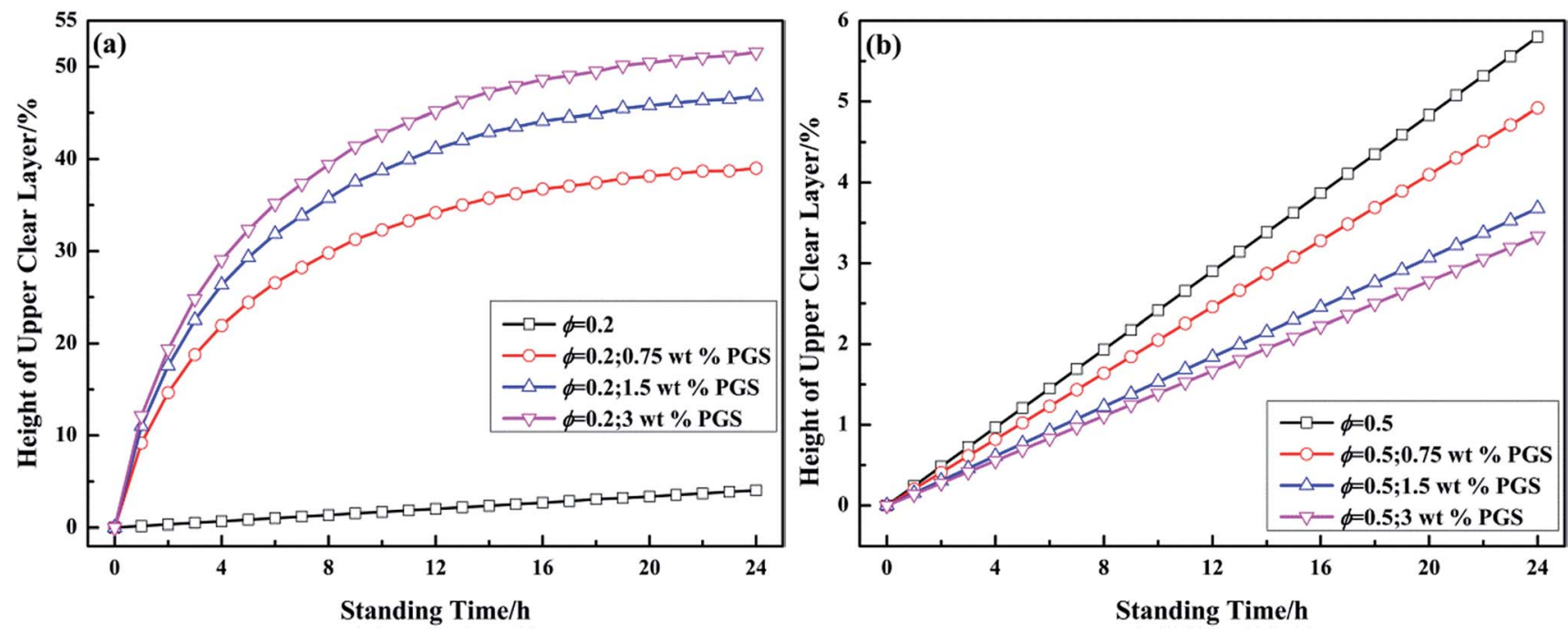

Fig. 8 Variations of the height of upper clear layer of emulsions at $\phi$ of 0.2 (a) and 0.5 (b) with different loadings of PGS with standing time. 


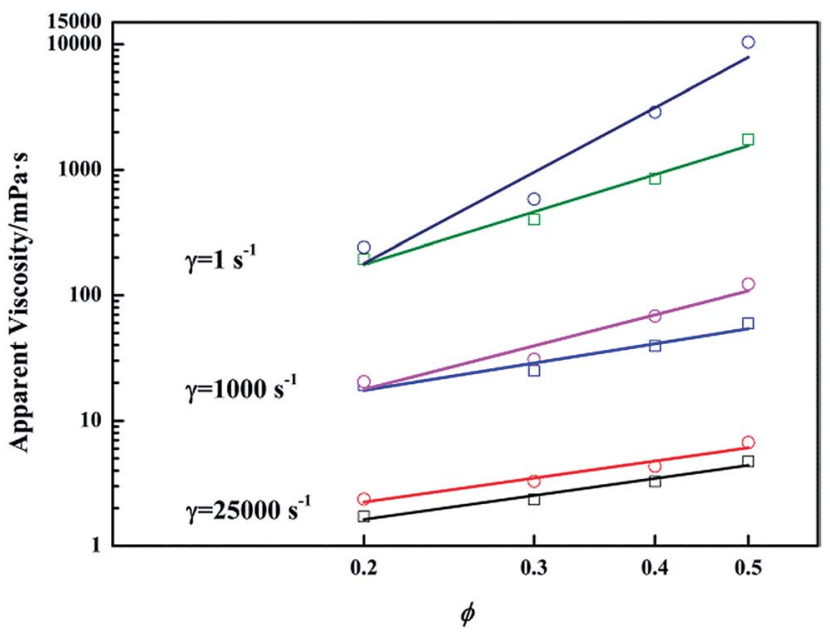

Fig. 9 Variations of apparent viscosities (measured at $1 \mathrm{~s}^{-1}, 1000 \mathrm{~s}^{-1}$ and $25000 \mathrm{~s}^{-1}$ ) with $\phi$ of pure emulsions (square symbols) and emulsions with 1.5 wt\% PGS (circular symbols).

Table 1 Interfacial tension of interfaces between paraffin and water with different loading of SPAN-80 and PGS

\begin{tabular}{lll}
\hline SPAN-80/wt $\%$ & PGS/wt $\%$ & $\begin{array}{l}\text { Interfacial } \\
\text { tension } / \mathrm{mN} \mathrm{m}^{-1}\end{array}$ \\
\hline 0 & 0 & 3.053 \\
0 & 0.05 & 1.665 \\
1 & 0 & 2.435 \\
1 & 0.05 & 2.679 \\
5 & 0 & 0.257 \\
5 & 0.05 & 0.274
\end{tabular}

emulsion with its impacts on interfacial properties. Table 1 presents the interfacial tension $\sigma$ of interfaces between paraffin and water with different loadings of SPAN-80 and PGS. It can be observed that PGS alone acts as a high-performance surfactant. At the addition of $0.05 \mathrm{wt} \%$, PGS decreases $\sigma$ of paraffin/water interface from $3.053 \mathrm{mN} \mathrm{m}^{-1}$ to $1.665 \mathrm{mN} \mathrm{m}^{-1}$. In contrast, the commonly-used water-in-oil emulsifier SPAN-80 only decreases $\sigma$ to $2.435 \mathrm{mN} \mathrm{m}^{-1}$ at $1 \mathrm{wt} \%$ loading. However, for interfaces adsorbed with SPAN-80, the addition of PGS increases $\sigma$, indicating that SPAN-80 and PGS are not synergistic. In the presence of SPAN-80, PGS exhibits different interfacial behaviors instead of arraying steadily at the paraffinwater phase boundary.

Interfacial rheology testing was utilized to further investigate the interfacial behaviors of PGS in the presence of SPAN80. Fig. 10a shows the dependence of interfacial complex modulus $\left|G^{*}\right|$ on frequency. It can be observed that for the interface of paraffin/water without any surfactants, $\left|G^{*}\right|$ grows linearly in the entire frequency range in double-logarithmic coordinate. After the addition of SPAN-80 and PGS, since surfactants create interfacial film by interfacial adsorption, $\left|G^{*}\right|$ of paraffin + SPAN-80/water interface and paraffin + SPAN80/water + PGS interface exhibit varying dependence on frequency. Before turning to linear growth at a critical frequency, $\left|G^{*}\right|$ grows progressively. The critical frequency in this measurement indicates the resistance of interfacial film to mechanical disturbance because when oscillation becomes sufficiently intense, the surfactants would lose stable arrangement and the film would thus be destroyed. By comparison, it is found that the complex interfacial film formed by both SPAN80 and PGS is more stable than the simple film formed by SPAN-80 alone. The former's critical frequency is $1.26 \mathrm{~Hz}$ while that of the latter is $0.47 \mathrm{~Hz}$. After film destruction, $\left|G^{*}\right|$ begins to reflect interactions of the two phases instead of film strength. The dependence of $\left|G^{*}\right|$ on frequency of all the interfaces becomes the same, which is well-proven by the almost equal slopes.

Fig. 10a shows that $\left|G^{*}\right|$ of the complex film is about one order of magnitude larger than that of the simple film,
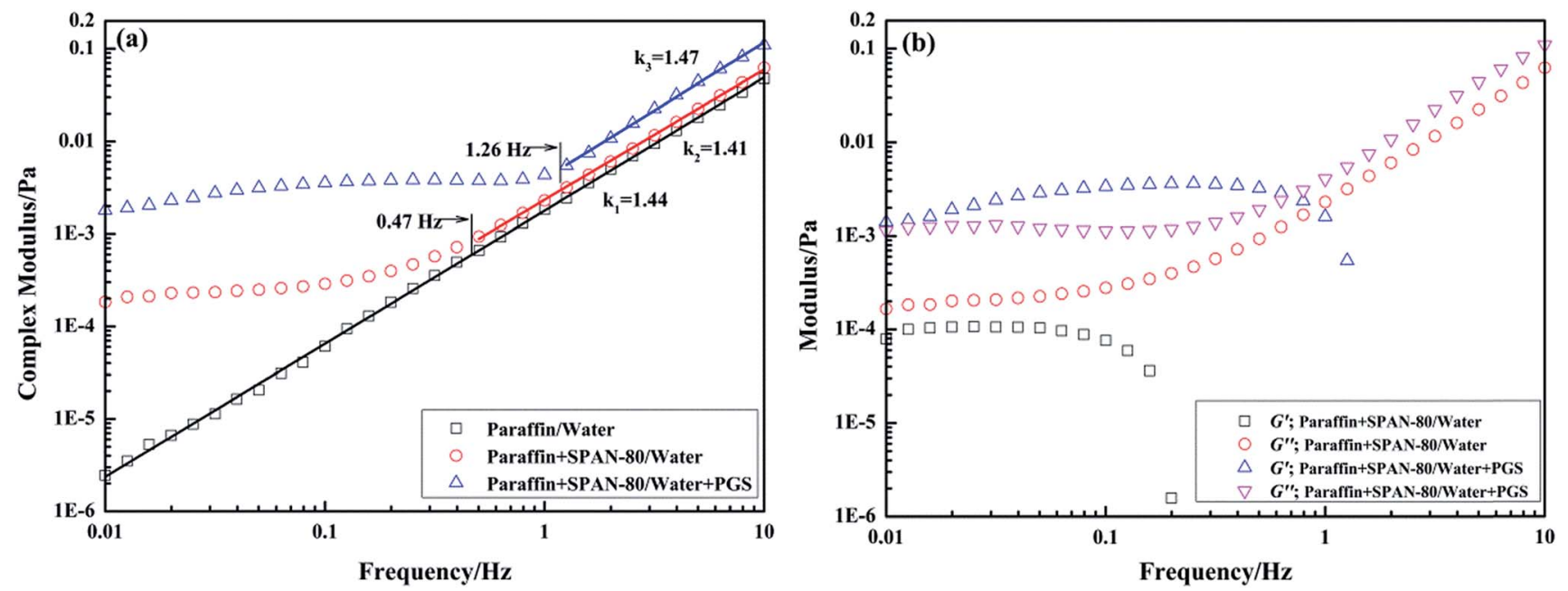

Fig. 10 Frequency dependencies of interfacial complex modulus $|G *|$ (a), elastic modulus $G^{\prime}$ and viscous modulus $G^{\prime \prime}$ (b) of paraffin/water interface, paraffin + SPAN-80/water interface and paraffin + SPAN-80/water + PGS interface. The addition of SPAN-80 in paraffin and PGS in water is $5 \mathrm{wt} \%$ and $1.5 \mathrm{wt} \%$ respectively. 


.

\footnotetext{
Fig. 11 Schematic illustration of simple interfacial film, complex interfacial film and states of PGS-loaded droplets under different standing times and shear magnitudes.
}

indicating that PGS strengthens film by interfacial accumulation. Further, from data of corresponding interfacial elastic modulus $G^{\prime}$ and viscous modulus $G^{\prime \prime}$ shown in Fig. 10b, it is known that for simple film, $G^{\prime \prime}$ predominates $G^{\prime}$ before film destruction. However, for complex film, $G^{\prime}$ predominates $G^{\prime \prime}$. Apparently, the addition of PGS makes the film gel-like. The interfacial rheology results are in close accordance with the droplet morphology. As observed in Fig. 7, droplets of pure emulsion are not totally circular owing to flexible film, while droplets of PGS-loaded emulsion have regular circular morphology, since the gelatinization of PGS makes film rigid and hard to deform.

\subsection{Probable mechanism}

According to experimental results above, it is clear that the mechano-responsive rheology modification of PGS on inverted emulsion is achieved by transforming water droplets from repulsive to attractive. As shown in Fig. 11, this sensitive attraction enables PGS-loaded droplets to change the extent of flocculation depending on standing time and shear magnitude, resulting in improved thixotropic and shear thinning performance. Such transition is attributed to interfacial accumulation of PGS, which is actualized by its rigid molecular structure and by hydrogen bonds between hydroxyls on PGS and SPAN-80. Since PGS increases interfacial tension, the short-range repulsion between emulsifier barriers of each droplet is weakened and droplets become prone to flocculation. The gelatinization of PGS on interfacial film also plays an important role as it not only stabilizes the film, but also increases resistance of droplets to deformation.

\subsection{Synergistic effect between PGS and OC}

The rheology modification of PGS on inverted emulsion enables PGS for use in clay-free IEDF. Nevertheless, though clay-free drilling fluid possesses many outstanding properties, ${ }^{\mathbf{4 1}}$ traditional IEDF containing OC is still the most commonly-used. Thus, it is necessary to investigate the performance of PGS in clay-loaded inverted emulsions. Table 2 presents rheology of emulsion at $\phi$ of 0.2 with different loading of OC (VG-Plus) and PGS. The rheology is evaluated under Bingham model which is given as

$$
\tau=\mathrm{YP}+\mathrm{PV} \times \gamma
$$

where $\tau$ is the shear stress, YP is the yield point, PV is the plastic viscosity and $\gamma$ is the shear rate. It is observed that PGS effectively increases LSRVs $\left(\phi_{6}, \phi_{3}\right)$ of emulsions with clay, which

Table 2 Rheology of emulsion at $\phi$ of 0.2 with different loading of OC (VG-Plus) and PGS under Bingham model

\begin{tabular}{|c|c|c|c|c|c|c|c|}
\hline VG-Plus/wt\% & PGS/wt\% & $\mathrm{AV} / \mathrm{mPa} \mathrm{s}$ & $\mathrm{PV} / \mathrm{mPa} \mathrm{s}$ & $\mathrm{YP} / \mathrm{Pa}$ & $\begin{array}{l}\mathrm{YP} / \mathrm{PV} / \mathrm{Pa} \\
(\mathrm{mPa} \mathrm{s})^{-1}\end{array}$ & $\phi_{6} / \phi_{3}$ & $\mathrm{Gel}_{10 \mathrm{~s}} / \mathrm{Gel}_{10 \mathrm{~min}} / \mathrm{Pa} \mathrm{Pa}{ }^{-1}$ \\
\hline 2 & 0 & 26 & 22 & 4.09 & 0.19 & $4 / 3$ & $1.44 / 1.44$ \\
\hline 2 & 1.5 & 33.5 & 25 & 8.69 & 0.35 & $6 / 5$ & $2.40 / 2.88$ \\
\hline 2 & 3 & 34 & 25 & 9.20 & 0.37 & $9 / 8$ & $3.84 / 4.80$ \\
\hline 4 & 0 & 38.5 & 30 & 8.69 & 0.29 & $7 / 6$ & $3.36 / 3.84$ \\
\hline 4 & 1.5 & 51 & 36 & 15.33 & 0.43 & $14 / 12$ & $5.76 / 6.72$ \\
\hline 4 & 3 & 53 & 37 & 16.35 & 0.44 & $17 / 14$ & $6.72 / 8.16$ \\
\hline
\end{tabular}


implies that the interfacial adsorption of PGS strengthens microstructures created by clay particles at paraffin/water interfaces, indicating a synergistic effect. As a result, the emulsion becomes more sensitive to shear, which is proven by increase of the plastic ratio (YP/PV). ${ }^{8}$ The improved progressive gel formation is also observed from the difference between initial gel strength $\left(\mathrm{Gel}_{10 \mathrm{~s}}\right)$ and final gel strength $\left(\mathrm{Gel}_{10 \mathrm{~min}}\right)$. In comparison, the use of PGS allows decreased clay loading. For example, emulsion loaded with $2 \mathrm{wt} \%$ clay and $1.5 \mathrm{wt} \%$ PGS has higher plastic ratio, but much lower AV and PV than emulsion loaded with $4 \mathrm{wt} \%$ clay. In this aspect, PGS is very desirable for traditional IEDFs.

\section{Conclusions}

In summary, the as-prepared PGS is capable of transforming water droplets of inverted emulsion from repulsive to attractive through interfacial accumulation. The sensitive attraction makes the emulsion more time-dependent and shear-sensitive by changing the extent of droplet flocculation under different mechanical conditions. The flocculation strengthens the interdroplet interaction and thus modifies the rheology of emulsion. As PGS is suitable for pure emulsion and clay-loaded emulsion, it can be utilized widely as a high-performance rheology modifier in clay-free and traditional IEDFs. To some extent, it can pave an innovative way to realize an absolute mechano-responsive "sol-gel" transition which enables IEDF to transform between a fluid with extremely low viscosity and a gel with high strength. Further work is in process.

\section{Conflicts of interest}

There are no conflicts to declare.

\section{Acknowledgements}

We would like to thank for the financial support from Foundation for Innovative Research Groups of the Natural Science Foundation of China (51221003), National Natural Science Foundation of China (51474231 and U1262201) and Foundation from Major Programs of China during 13th Five-Year Plan Period (2016ZX05022-001-001-001, 2016ZX05040-001-002 and 2016ZX05020-004) for this work. We thank Hu Yuanyuan in Shandong University for taking cryo-TEM images.

\section{References}

1 F. Growcock and T. Harvey, Drilling Fluids, in Drilling Fluids Processing Handbook, Gulf Professional Publishing, Burlington, 2005, ch. 2, pp. 15-68.

2 F. T. G. Dias, R. R. Souza and E. F. Lucas, Influence of modified starches composition on their performance as fluid loss additives in invert-emulsion drilling fluids, Fuel, 2015, 140, 711-716.

3 J. Wang, X. Zhang, H. Cao, K. Guo and W. Liu, Application of an environmentally-friendly synthetic base drilling fluid to horizontal drilling of shale gas wells, 2013, vol. 33, pp. 82-85.
4 K. Song, Q. Wu, M. Li, S. Ren, L. Dong, X. Zhang, T. Lei and Y. Kojima, Water-based bentonite drilling fluids modified by novel biopolymer for minimizing fluid loss and formation damage, Colloids Surf., A, 2016, 507, 58-66.

5 Y. An, G. Jiang, Y. Qi, Q. Ge, L. Zhang and Y. Ren, Synthesis of nano-plugging agent based on AM/AMPS/NVP terpolymer, $J$. Pet. Sci. Eng., 2015, 135(suppl. C), 505-514.

6 Y. Xuan, G. Jiang, Y. Li, J. Wang and H. Geng, Inhibiting effect of dopamine adsorption and polymerization on hydrated swelling of montmorillonite, Colloids Surf., A, 2013, 422(suppl. C), 50-60.

7 N. Alimohammadi, S. R. Shadizadeh and I. Kazeminezhad, Removal of cadmium from drilling fluid using nanoadsorbent, Fuel, 2013, 111(suppl. C), 505-509.

8 R. Caenn, H. C. H. Darley and G. R. Gray, The Rheology of Drilling Fluids, in Composition and Properties of Drilling and Completion Fluids, Gulf Professional Publishing, Boston, 6th edn, 2011, ch. 5, pp. 179-269.

9 W. Weng, J. B. Beck, A. M. Jamieson and S. J. Rowan, Understanding the Mechanism of Gelation and StimuliResponsive Nature of a Class of Metallo-Supramolecular Gels, J. Am. Chem. Soc., 2006, 128(35), 11663-11672.

10 Y. Sagara and N. Tamaoki, Mechanoresponsive luminescence and liquid-crystalline behaviour of a cyclophane featuring two 1,6-bis(phenylethynyl)pyrene groups, RSC Adv., 2017, 7(74), 47056-47062.

11 B. Werner, V. Myrseth and A. Saasen, Viscoelastic properties of drilling fluids and their influence on cuttings transport, $J$. Pet. Sci. Eng., 2017, 156(suppl. C), 845-851.

12 S. Sayindla, B. Lund, J. D. Ytrehus and A. Saasen, Holecleaning performance comparison of oil-based and waterbased drilling fluids, J. Pet. Sci. Eng., 2017, 159(suppl. C), 49-57.

13 S. R. Derkach, Rheology of emulsions, Adv. Colloid Interface Sci., 2009, 151(1-2), 1-23.

14 T. G. Mason, New fundamental concepts in emulsion rheology, Curr. Opin. Colloid Interface Sci., 1999, 4(3), 231238.

15 A. Koenig, P. Hébraud and P. Perrin, Preparation and Rheological Properties of Emulsion Gels, Langmuir, 2002, 18(16), 6458-6461.

16 T. G. Mason, M.-D. Lacasse, G. S. Grest, D. Levine, J. Bibette and D. A. Weitz, Osmotic pressure and viscoelastic shear moduli of concentrated emulsions, Phys. Rev. E, 1997, 56(3), 3150-3166.

17 R. Pal, Y. Yan and J. Masliyah, Rheology of Emulsions, in Emulsions, American Chemical Society, 1992, vol. 231, pp. 131-170.

$18 \mathrm{~J}$. Hermoso, F. Martinez-Boza and C. Gallegos, Influence of aqueous phase volume fraction, organoclay concentration and pressure on invert-emulsion oil muds rheology, J. Ind. Eng. Chem., 2015, 22, 341-349.

$19 \mathrm{~J}$. Hermoso, F. Martinez-Boza and C. Gallegos, Influence of viscosity modifier nature and concentration on the viscous flow behaviour of oil-based drilling fluids at high pressure, Appl. Clay Sci., 2014, 87, 14-21. 
20 D. Zhou, Z. Zhang, J. Tang, F. Wang and L. Liao, Applied properties of oil-based drilling fluids with montmorillonites modified by cationic and anionic surfactants, Appl. Clay Sci., 2016, 121-122, 1-8.

21 G. Zhuang, Z. Zhang, J. Sun and L. Liao, The structure and rheology of organo-montmorillonite in oil-based system aged under different temperatures, Appl. Clay Sci., 2016, 124-125, 21-30.

22 G. Zhuang, Z. Zhang, M. Fu, X. Ye and L. Liao, Comparative study on the use of cationic-nonionic-organomontmorillonite in oil-based drilling fluids, Appl. Clay Sci., 2015, 116(suppl. C), 257-262.

23 G. Zhuang, Z. Zhang, M. Fu, X. Ye and L. Liao, Comparative study on the use of cationic-nonionic-organomontmorillonite in oil-based drilling fluids, Appl. Clay Sci., 2015, 116-117, 257-262.

24 M. N. Lee, H. K. Chan and A. Mohraz, Characteristics of Pickering Emulsion Gels Formed by Droplet Bridging, Langmuir, 2012, 28(6), 3085-3091.

25 H. Kim, K. Kim, H.-R. Lee, H. Jo, D.-w. Jeong, J. Ryu, D.-G. Gweon and S. Q. Choi, Formation of stable adhesive water-in-oil emulsions using a phospholipid and cosurfactants, J. Ind. Eng. Chem., 2017, 55, 198-203.

26 F. M. Menger and C. A. Littau, Gemini surfactants: a new class of self-assembling molecules, J. Am. Chem. Soc., 1993, 115(22), 10083-10090.

27 M. Dreja and B. Tieke, Polymerization of Styrene in Ternary Microemulsion Using Cationic Gemini Surfactants, Langmuir, 1998, 14(4), 800-807.

28 K. Sakai, K. Ohno, K. Nomura, T. Endo, K. Sakamoto, H. Sakai and M. Abe, $\alpha$-Gel Formation by Amino AcidBased Gemini Surfactants, Langmuir, 2014, 30(26), 76547659.

29 D. Xie, D. You, S. Ying, B. Song and J. Tian, Viscoelastic properties of supramolecular gemini-like surfactant solutions in the absence of inorganic salts, RSC Adv., 2017, 7(76), 48120-48126.

30 M. N. Maithufi, D. J. Joubert and B. Klumperman, Application of Gemini Surfactants as Diesel Fuel Wax Dispersants, Energy Fuels, 2011, 25(1), 162-171.

31 C. McGregor, C. Perrin, M. Monck, P. Camilleri and A. J. Kirby, Rational Approaches to the Design of Cationic
Gemini Surfactants for Gene Delivery, J. Am. Chem. Soc., 2001, 123(26), 6215-6220.

32 Y.-j. Guo, J.-x. Liu, X.-m. Zhang, R.-s. Feng, H.-b. Li, J. Zhang, X. Lv and P.-y. Luo, Solution Property Investigation of Combination Flooding Systems Consisting of Gemini-Nonionic Mixed Surfactant and Hydrophobically Associating Polyacrylamide for Enhanced Oil Recovery, Energy Fuels, 2012, 26(4), 2116-2123.

33 E. Hablot, B. Donnio, M. Bouquey and L. Avérous, Dimer acid-based thermoplastic bio-polyamides: reaction kinetics, properties and structure, Polymer, 2010, 51(25), 5895-5902.

34 B. C. Leopércio, P. R. de Souza Mendes and G. G. Fuller, Growth Kinetics and Mechanics of Hydrate Films by Interfacial Rheology, Langmuir, 2016, 32(17), 4203-4209.

35 O. Mengual, G. Meunier, I. Cayré, K. Puech and P. Snabre, TURBISCAN MA 2000: multiple light scattering measurement for concentrated emulsion and suspension instability analysis, Talanta, 1999, 50(2), 445-456.

36 K. J. Park, M. Kim, S. Seok, Y.-W. Kim and D. H. Kim, Quantitative analysis of cyclic dimer fatty acid content in the dimerization product by proton NMR spectroscopy, Spectrochim. Acta, Part A, 2015, 149, 402-407.

37 I. S. Chronakis and P. Alexandridis, Rheological Properties of Oppositely Charged Polyelectrolyte-Surfactant Mixtures: Effect of Polymer Molecular Weight and Surfactant Architecture, Macromolecules, 2001, 34(14), 5005-5018.

$38 \mathrm{~T}$. Tadros, Viscoelastic properties of sterically stabilised emulsions and their stability, Adv. Colloid Interface Sci., 2015, 222, 692-708.

39 F. Y. Ushikubo and R. L. Cunha, Stability mechanisms of liquid water-in-oil emulsions, Food Hydrocolloids, 2014, 34, 145-153.

40 H. Tanaka, S. Yamamoto, A. Nakamura, Y. Nakashoji, N. Okura, N. Nakamoto, K. Tsukagoshi and M. Hashimoto, Hands-Off Preparation of Monodisperse Emulsion Droplets Using a Poly(dimethylsiloxane) Microfluidic Chip for Droplet Digital PCR, Anal. Chem., 2015, 87(8), 4134-4143.

41 X. Zhang, G. Jiang, Y. Xuan, L. Wang and X. Huang, Associating Copolymer Acrylamide/ Diallyldimethylammonium Chloride/Butyl Acrylate/2Acrylamido-2-Methylpropanesulfonic Acid as a Tackifier in Clay-Free and Water-Based Drilling Fluids, Energy Fuels, 2017, 31(5), 4655-4662. 